\title{
Controllability for nonlinear evolution equations with monotone operators
}

\author{
Yong Han Kang ${ }^{1}$, Jin-Mun Jeong ${ }^{2 *}$ and Hyun-Hee Rho ${ }^{2}$
}

${ }^{\text {"Correspondence: }}$

jmjeong@pknu.ac.kr

${ }^{2}$ Department of Applied

Mathematics, Pukyong National

University, Busan, 608-737, Korea

Full list of author information is

available at the end of the article

\begin{abstract}
In this paper, we investigate the approximate controllability for nonlinear evolution equations with monotone operators and nonlinear controllers according to monotone operator theory. We also give the regularity for the nonlinear equation. Finally, an example, to which our main result can be applied, is given.
\end{abstract}

MSC: $35 F 25 ; 93 C 20$

Keywords: nonlinear evolution equation; monotone operator; approximate controllability; regularity; hemicontinuous

\section{Introduction}

In this paper, we deal with the approximate controllability for the semilinear equation in a Hilbert space $H$ as follows:

$$
\left\{\begin{array}{l}
x^{\prime}(t)+A x(t)+f(t, x(t), u(t))+B u(t)=0, \quad 0<t \leq T, \\
x(0)=x_{0} .
\end{array}\right.
$$

In (1.1), the principal operator $-A$ generates an analytic semigroup $S(t)$. Let $U$ be a Hilbert space of control variables, and let $B$ be a linear (or nonlinear) operator from $U$ to $H$, which is called a controller.

First, we consider the following initial value problem of a semilinear equation:

$$
\left\{\begin{array}{l}
x^{\prime}(t)+A x(t)+f(t, x(t))=h(t), \quad 0<t \leq T, \\
x(0)=x_{0} .
\end{array}\right.
$$

If $A: D(A) \subset H \rightarrow H$ is an unbounded operator, Di Blasio et al. [1] proved $L^{2}$-regularity for a retarded linear system in Hilbert spaces, and Jeong [2] (also see [3]) considered the control problem for retarded linear systems with $L^{1}$-valued controller and more general Lipschitz continuity of nonlinear terms.

For the theory of monotone operators, there are many literature works; for example, see Lions [4], Stampacchia [5], Browder [6], and the references cited therein. Kenmochi [7] derived new results on monotone operator equations, and Ouchi [8] proved the analyticity of solutions of semilinear parabolic differential equations with monotone nonlinearity. For the existence of solutions for a class of nonlinear evolution equations with monotone perturbations, one can refer to [9-11]. We refer to Pascali and Sburlan [12], Morosanu [13]

\section{Springer}

○2013 Kang et al.; licensee Springer. This is an Open Access article distributed under the terms of the Creative Commons Attribution License (http://creativecommons.org/licenses/by/2.0), which permits unrestricted use, distribution, and reproduction in any medium, provided the original work is properly cited. 
to see the applications of nonlinear mapping of monotone type and nonlinear evolution equations. The classical solutions of (1.2) were obtained by Kato [14] under the monotonicity condition on the nonlinear term $f$ as an operator from $[0, T] \times H$ to $H$.

In the first part of this note, we apply results of [14] to find $L^{2}$-regularity of solutions in the wider sense of (1.2) under the more general monotonicity of a nonlinear operator $f$ from $\mathbb{R} \times V$ to $V^{*}$, which is related to the results of Tanabe [15, Theorem 6.6.2].

Next, we extend and develop control problems on this topic. In recent years, as for the controllability for semilinear differential equations with Lipschitz continuity of a nonlinear operator $f$, Naito [16] and [17-19] proved the approximate controllability under the range conditions of the controller $B$. However, we can find few articles which extend the known general controllability problems to nonlinear evolution equation (1.1) with monotone operators and nonlinear controllers.

In this paper, based on the regularity for solutions of equation (1.2), we obtain the approximate controllability for nonlinear evolution equation (1.1) with monotone operators and nonlinear controllers.

The paper is organized as follows. In Section 2, we explain several notations of this paper and state results about $L^{2}$-regularity for linear equations in the sense of $[1,15,20]$. In Section 3, we give the regularity for nonlinear equation (1.2). In Section 4, we obtain the approximate controllability for nonlinear evolution equation (1.1) with hemicontinuous monotone operators by using the theory of monotone operators. In the end, an example is provided to illustrate the application of the obtained results.

\section{Preliminaries}

If $H$ is identified with its dual space, we may write $V \subset H \subset V^{*}$ densely and the corresponding injections are continuous. The norm on $V, H$ and $V^{*}$ will be denoted by $\|\cdot\|,|\cdot|$ and $\|\cdot\|_{*}$, respectively. The duality pairing between the element $v_{1}$ of $V^{*}$ and the element $v_{2}$ of $V$ is denoted by $\left(v_{1}, v_{2}\right)$, which is the ordinary inner product in $H$ if $v_{1}, v_{2} \in H$.

For $l \in V^{*}$, we denote $(l, v)$ by the value $l(v)$ of $l$ at $v \in V$. The norm of $l$ as element of $V^{*}$ is given by

$$
\|l\|_{*}=\sup _{v \in V} \frac{|(l, v)|}{\|v\|} .
$$

Therefore, we assume that $V$ has a stronger topology than $H$ and, for brevity, we may regard that

$$
\|u\|_{*} \leq|u| \leq\|u\|, \quad \forall u \in V .
$$

Let $a(\cdot, \cdot)$ be a bounded sesquilinear form defined in $V \times V$ and satisfying Gårding's inequality

$$
\operatorname{Re} a(u, u) \geq \omega_{1}\|u\|^{2}-\omega_{2}|u|^{2},
$$

where $\omega_{1}>0$ and $\omega_{2}$ is a real number. Let $A$ be an operator associated with this sesquilinear form:

$$
(A u, v)=a(u, v), \quad u, v \in V .
$$


Then $-A$ is a bounded linear operator from $V$ to $V^{*}$ by the Lax-Milgram theorem. The realization of $A$ in $H$, which is the restriction of $A$ to

$$
D(A)=\{u \in V: A u \in H\},
$$

is also denoted by $A$. It is well known that $A$ is positive definite and self-adjoint and generates an analytic semigroup $S(t)$ in both $H$ and $V^{*}$. From the following inequalities:

$$
\omega_{1}\|u\|^{2} \leq \operatorname{Re} a(u, u)+\omega_{2}|u|^{2} \leq C\left|A u\left\|\left.u\left|+\omega_{2}\right| u\right|^{2} \leq \max \left\{C, \omega_{2}\right\}\right\| u \|_{D(A)}\right| u \mid,
$$

where

$$
\|u\|_{D(A)}=\left(|A u|^{2}+|u|^{2}\right)^{1 / 2}
$$

is the graph norm of $D(A)$, it follows that there exists a constant $C_{0}>0$ such that

$$
\|u\| \leq C_{0}\|u\|_{D(A)}^{1 / 2}|u|^{1 / 2}
$$

Thus we have the following sequence:

$$
D(A) \subset V \subset H \subset V^{*} \subset D(A)^{*},
$$

where each space is dense in the next one, which is continuous injection.

Lemma 2.1 With notations (2.3), (2.4), we have

$$
\begin{aligned}
& \left(V, V^{*}\right)_{1 / 2,2}=H, \\
& (D(A), H)_{1 / 2,2}=V,
\end{aligned}
$$

where $\left(V, V^{*}\right)_{1 / 2,2}$ denotes the real interpolation space between $V$ and $V^{*}$ (Section 2.4 of [21] or [22]).

If $X$ is a Banach space, $L^{2}(0, T ; X)$ is the collection of all strongly measurable square integrable functions from $(0, T)$ into $X$, and $W^{1,2}(0, T ; X)$ is the set of all absolutely continuous functions on $[0, T]$ such that their derivative belong to $L^{2}(0, T ; X) . C([0, T] ; X)$ will denote the set of all continuous functions from $[0, T]$ into $X$ with the supremum norm. If $X$ and $Y$ are two Banach spaces, $\mathcal{L}(X, Y)$ is the collection of all bounded linear operators from $X$ into $Y$, and $\mathcal{L}(X, X)$ is simply written as $\mathcal{L}(X)$. Here, we note that by using interpolation theory, we have

$$
L^{2}(0, T ; V) \cap W^{1,2}\left(0, T ; V^{*}\right) \subset C([0, T] ; H) .
$$

First, we consider the following linear system:

$$
\left\{\begin{array}{l}
x^{\prime}(t)+A x(t)=k(t), \\
x(0)=x_{0}
\end{array}\right.
$$


By virtue of Theorem 3.3 of [1] (or Theorem 3.1 of $[2,15]$ ), we have the following result on the corresponding linear equation of (2.6).

Lemma 2.2 (1) For $x_{0} \in V=(D(A), H)_{1 / 2,2}$ (see Lemma 2.1) and $k \in L^{2}(0, T ; H), T>0$, there exists a unique solution $x$ of (2.6) belonging to

$$
L^{2}(0, T ; D(A)) \cap W^{1,2}(0, T ; H) \subset C([0, T] ; V)
$$

and satisfying

$$
\|x\|_{L^{2}(0, T ; D(A)) \cap W^{1,2}(0, T ; H)} \leq C_{1}\left(\left\|x_{0}\right\|+\|k\|_{L^{2}(0, T ; H)}\right),
$$

where $C_{1}$ is a constant depending on $T$.

(2) Let $x_{0} \in H$ and $k \in L^{2}\left(0, T ; V^{*}\right), T>0$. Then there exists a unique solution $x$ of (2.6) belonging to

$$
L^{2}(0, T ; V) \cap W^{1,2}\left(0, T ; V^{*}\right) \subset C([0, T] ; H)
$$

and satisfying

$$
\|x\|_{L^{2}(0, T ; V) \cap W^{1,2}\left(0, T ; V^{*}\right)} \leq C_{1}\left(\left|x_{0}\right|+\|k\|_{L^{2}\left(0, T ; V^{*}\right)}\right),
$$

where $C_{1}$ is a constant depending on $T$.

Throughout this paper, strong convergence is denoted by ' $\rightarrow$ ' and weak convergence by ' $\rightarrow$ '.

Definition 2.1 Let $X$ and $Y$ be Banach spaces and $L$ be a mapping from $X$ into $Y$. The domain $D(L)$ of $L$ is assumed to be convex. $L$ is called hemicontinuous if $L\left((1-\lambda) x_{1}+\lambda x_{2}\right)$ for any $x_{1}, x_{2} \in D(L)$ is continuous in $0 \leq \lambda \leq 1$ in weak topology of $Y$.

The linear operator is obviously hemicontinuous.

Definition 2.2 Let $X$ and $Y$ be Banach spaces and $L$ be a single-valued mapping from $X$ into $Y . L$ is called demicontinuous if $x_{n} \in D(L)$ and $x_{n} \rightarrow x \in D(L)$ imply that $L x_{n} \rightarrow L x$.

Definition 2.3 Let $L$ be a mapping from a Banach space $X$ into its conjugate space $X^{*}$. $L$ is said to be pseudo-monotone if the following condition is satisfied. If $x_{i}$ is a directed family of points, contained in $D(L)$, which converges weakly to an element $x$ of $D(L)$ and if $\lim \sup \left(L x_{i}, x_{i}-x\right) \leq 0$, then $\liminf \left(L x_{i}, x_{i}-y\right) \geq(L x, y)$ for all $y \in D(L)$.

Definition 2.4 Let $L$ be a generally multi-valued mapping from a Hilbert space $X$ into itself. If

$$
(\hat{x}-\hat{y}, x-y) \geq 0
$$


for all $x, y \in D(L), \hat{x} \in L x$ and $\hat{y} \in L y$, then $L$ is called a monotone operator. Sometimes $L$ is also called a monotone operator if

$$
(\hat{x}-\hat{y}, x-y) \leq 0
$$

holds instead of (2.9).

Definition 2.5 A real-valued continuous function $j$ is called a gauge function defined on $[0, \infty)$ if it is strictly monotone increasing and satisfies $j(0)=0$ and $\lim _{r \rightarrow \infty} j(r)=\infty$.

Let $X$ be a Banach space and $X^{*}$ its conjugate. For any $x \in X$, we set

$$
\mathcal{F}(x)=\left\{x^{*} \in X^{*}:\left(x^{*}, x\right)=\left\|x^{*}\right\|_{*}\|x\|,\left\|x^{*}\right\|_{*}=j(\|x\|)\right\} .
$$

The multi-valued operator $\mathcal{F}: X \rightarrow X^{*}$ is called the duality mapping of $X$ with a gauge function $j$.

Let us denote by $\Lambda$ the operator determined by an inner product $((\cdot, \cdot))$ on $V:(\Lambda x, y)=$ $((x, y))$. Then it is immediate that $\Lambda$ is a duality mapping from $V$ into $V^{*}$ with a gauge function $j(r)=r$. It is also known that the duality mapping is monotone and hemicontinuous, and hence it is pseudo-monotone.

Lemma 2.3 We have briefly explained the theory of monotone operators (see [15, Section 6.6]).

(1) In Definition 2.3 above, $\lim \left(L x_{i}, x_{i}-x\right)=0$ is seen by taking $x=y$.

(2) Hemicontinuous monotone mappings from a Banach space $X$ into $X^{*}$ are pseudo-monotone.

(3) Let $X$ be a reflexive Banach space, and let both $X$ and $X^{*}$ be strictly convex. Further, let $M \subset X \times X^{*}$ be monotone and $\Lambda$ be a duality mapping from $X$ into $X^{*}$. If $R(M+\Lambda)=X^{*}$, then $M$ is maximal monotone.

(4) Let $X$ be a reflexive Banach space and $L$ be a closed monotone linear operator from $X$ and $X^{*}$. If the dual operator $L^{*}$ is monotone, then $L$ is maximal monotone.

(5) Let $X$ be a reflexive Banach space, $M \subset X \times X^{*}$ be maximal monotone and $L$ be a pseudo-monotone bounded mapping from $D(L)=X$ into $X^{*}$. If there exists $\left[x_{0}, f_{0}\right] \in M$ such that

$$
\lim _{\|x\| \rightarrow \infty}\left(L x+f_{0}, x-x_{0}\right) /\|x\|=\infty
$$

then $R(M+L)=X^{*}$, that is, for every $f \in X^{*},(M+L) x \ni f$ has a solution $x \in D(M)$.

The following inequality is referred to as Young's inequality.

Lemma 2.4 (Young's inequality) Let $a>0, b>0$ and $1 / p+1 / q=1$, where $1 \leq p<\infty$ and $1<q<\infty$. Then, for every $\lambda>0$, one has

$$
a b \leq \frac{\lambda^{p} a^{p}}{p}+\frac{b^{q}}{\lambda^{q} q} .
$$


Lemma 2.5 Let $H$ be a Hilbert space and $V$ be a reflexive Banach space. Suppose that $V$ is a dense subspace of $H$ and that $V$ has a stronger topology than $H$. Therefore, $V \subset H \subset V^{*}$. Let $T>0$ and $X=L^{p}(0, T ; V)$ with $2 \leq p<\infty$. Then the operator $L$ defined by

$$
\left\{\begin{array}{l}
D(L)=\left\{x \in X: x^{\prime} \in X^{*}, x(0)=0\right\} \\
L x=x^{\prime} \text { for each } x \in D(L)
\end{array}\right.
$$

is maximal monotone linear.

Proof Since $X^{*}=L^{q}\left(0, T ; V^{*}\right)$ with $q=p /(p-1)$, noting that $x^{\prime}(t) \in V^{*}$ and by Young's inequality, we have

$$
\begin{aligned}
|x(t)|^{2} & =\int_{0}^{t}(d / d s)|x(s)|^{2} d s=\int_{0}^{t} 2 \operatorname{Re}\left(x^{\prime}(s), x(s)\right) d s \\
& \leq 2^{q} \int_{0}^{t}\left\|x^{\prime}(s)\right\|^{q} / q d s+\int_{0}^{t}\|x(s)\|^{p} / p d s,
\end{aligned}
$$

so that $x$ belongs to $C([0, T] ; H)$. Therefore, we find that $x(0)$ is well defined as an element of $H$. It is easily shown that $L$ is a closed linear operator from $X$ into $X^{*}$ and $D(L)$ is dense in $X$. Further, since

$$
(L x, x)=\left(x^{\prime}, x\right)=|x(T)|^{2} / 2 \geq 0,
$$

we know that $L$ is monotone. It is also easily seen that the adjoint operator of $L$ is given by

$$
\left\{\begin{array}{l}
D\left(L^{*}\right)=\left\{x \in X: x^{\prime} \in X^{*}, x(T)=0\right\} \\
L^{*} x=-x^{\prime} \quad \text { for each } x \in D\left(L^{*}\right)
\end{array}\right.
$$

Hence, $L^{*}$ is also monotone. Therefore, from (4) of Lemma 2.3, it is concluded that $L$ is maximal monotone linear.

\section{Nonlinear equations}

We consider the following initial value problem of a semilinear equation:

$$
\left\{\begin{array}{l}
x^{\prime}(t)+A x(t)+f(t, x(t))=h(t), \quad 0<t \leq T, \\
x(0)=x_{0} .
\end{array}\right.
$$

The following result is due to Kato [7] (or [15, Theorem 6.6.1]).

Lemma 3.1 Letf be a demicontinuous bounded mapping from $[0, T] \times H$ into $H$. Assume that $f(t, \cdot)$ is monotone for each $t \in[0, T]$ :

$$
(f(t, x)-f(t, y), x-y)>0 .
$$

Assume further that $-A$ is a generator of a contraction semigroup $S(t)(t \geq 0)$. Then, for any $\left(x_{0}, h\right) \in H \times C([0, T] ; H)$, there exists a solution $x \in C([0, T] ; H)$ of the integral equation

$$
x(t)=S(t) x_{0}-\int_{0}^{t} S(t-s)\{f(s, x(s))-h(s)\} d s, \quad 0 \leq t \leq T,
$$


corresponding to (3.1), and it is unique. Let $x_{1}$ and $x_{2}$ be the solutions with initial values $x_{01}$ and $x_{02}$, respectively. Then the estimate

$$
\left|x_{1}(t)-x_{2}(t)\right| \leq\left|x_{01}-x_{02}\right|
$$

holds on $0 \leq t \leq T$. Hence, the mapping which carries the initial value $x_{0}$ to the solution $x$ is a continuous mapping from $H$ into $C([0, T] ; H)$.

Next, we apply Lemma 3.1 to find a solution in the wider sense of (3.1) under somewhat different assumptions. Concerning the nonlinear mapping $f$, assume the following hypothesis.

Assumption (F) The mapping $f$ is demicontinuous bounded from $[0, T] \times H$ into $V^{*}$, and $f(t, \cdot)$ for each $t$ is monotone as a mapping from $V$ into $V^{*}$.

The following theorem is a part of Theorem 6.6.2 due to Tanabe [15].

Theorem 3.1 Let Assumption (F) be satisfied, and let the assumptions on the principal operator A stated in Section 2 be satisfied. Assume that $x_{0}$ is an arbitrary element of $H$ and $h \in L^{2}\left(0, T ; V^{*}\right)$. Then there exists a solution $x \in L^{2}(0, T ; V)$, satisfying $x^{\prime} \in L^{2}\left(0, T ; V^{*}\right)$, of

$$
\left\{\begin{array}{l}
x^{\prime}(t)+A x(t)+f(t, x(t))=h(t), \quad 0<t \leq T \\
x(0)=x_{0}
\end{array}\right.
$$

and it is unique. Moreover, there exists a constant $C_{2}$ such that

$$
\|x\|_{L^{2}(0, T ; V) \cap W^{1,2}\left(0, T ; V^{*}\right)} \leq C_{2}\left(\left|x_{0}\right|+\|h\|_{L^{2}\left(0, T ; V^{*}\right)}\right),
$$

where $C_{2}$ is a constant depending on $T$ and the mapping

$$
H \times L^{2}\left(0, T ; V^{*}\right) \ni\left(x_{0}, h\right) \mapsto x \in L^{2}(0, T ; V) \cap W^{1,2}\left(0, T ; V^{*}\right) \subset C([0, T] ; H)
$$

is Lipschitz continuous.

Proof As far as the existence of the solution is concerned, we may put $x_{0}=0$. By Lemma 2.5, the operator defined by

$$
\left\{\begin{array}{l}
D(L)=\left\{x \in L^{2}(0, T ; V): x^{\prime} \in L^{2}\left(0, T ; V^{*}\right), x(0)=0\right\} \\
L x=x^{\prime}, \quad \forall x \in D(L),
\end{array}\right.
$$

is a maximal monotone linear operator from $L^{2}(0, T ; V)$ into $L^{2}\left(0, T ; V^{*}\right)$. Let us write $(A x)(t)=A x(t)$ and $(F x)(t)=f(t, x(t))$ for each $x \in L^{2}(0, T ; V)$. Then both $A$ and $F$ are monotone operators from $L^{2}(0, T ; V)$ into $L^{2}\left(0, T ; V^{*}\right)$ and $D(L) \subset D(F)$. Since we assumed $x_{0}=0$, equation (3.2) is equivalent to

$$
(L+F+A) x=h .
$$


Note that $L+F$ is monotone, if it is shown to be maximal monotone, assumption (5) of Lemma 2.3 is satisfied with $M=L+F, L=A$ and $x_{0}=0$. Then we have $R(L+F+A)=$ $L^{2}\left(0, T ; V^{*}\right)$, which implies the existence of the solution. Thus, from now on, we prove the maximal monotonicity of $L+F$. Since $\Lambda$ determined by an inner product $((\cdot, \cdot))$ on $V$ is a duality mapping from $V$ into $V^{*}$, as seen under Definition 2.5, to see the maximal monotonicity of $L+F$, on account of (3) of Lemma 2.3, it is enough to verify that $R(L+F+$ $\Lambda)=L^{2}\left(0, T ; V^{*}\right)$.

Let $h$ be an arbitrary element of $L^{2}\left(0, T ; V^{*}\right), h_{n} \in C([0, T] ; H)$ and $h_{n} \rightarrow h \operatorname{in} L^{2}\left(0, T ; V^{*}\right)$. Since $\Lambda$ is positive definite and self-adjoint in both $H$ and $V^{*}$, the domain $\Lambda^{1 / 2}$ coincides with $V$ and $H$, respectively. Hence, $I_{n}=\left(I+n^{-1} \Lambda^{1 / 2}\right)^{-1}$ is a contraction operator in both $H$ and $V$, and it converges strongly to $I$ as $n \rightarrow \infty$. It is also easy to see that $\left(I_{n} f, g\right)=\left(f, I_{n} g\right)$ holds for $f \in V^{*}$ and $g \in H$. Let us define $f_{n}(t, x)=I_{n} f(t, x)$. Then the mapping $f_{n}$ satisfies the assumption for $f$ in Lemma 3.1. Hence, Lemma 3.1 can be applied to the initial value problem

$$
\left\{\begin{array}{l}
x_{n}^{\prime}(t)+\Lambda x_{n}(t)+f_{n}\left(t, x_{n}(t)\right)-h_{n}(t)=0, \\
x(0)=0
\end{array}\right.
$$

Let us denote the semigroup generated by $\Lambda$ by $T(t)$. Then it ensures the existence of a solution $x \in C([0, T] ; H)$ of the equation

$$
x_{n}(t)+\int_{0}^{t} T(t-s)\left(f_{n}\left(s, x_{n}(s)\right)-h_{n}(s)\right) d s=0 .
$$

Multiplying by $x_{n}(t)$ on (3.3) and integrating over $[0, t]$, we have

$$
\frac{1}{2}\left|x_{n}(t)\right|^{2}+\int_{0}^{t}\left\|x_{n}(s)\right\|^{2} d s=\int_{0}^{t}\left(h_{n}(s)-f_{n}\left(s, x_{n}(s)\right), x_{n}(s)\right) d s .
$$

By using Young's inequality and the monotonicity of $f$, the following holds:

$$
\begin{aligned}
& \frac{1}{2}\left|x_{n}(t)\right|^{2}+\int_{0}^{t}\left\|x_{n}(s)\right\|^{2} d s \\
&=-\int_{0}^{t}\left(f_{n}\left(s, x_{n}(s)\right)-f_{n}(s, 0), x_{n}(s)\right) d s \\
& \quad-\int_{0}^{t}\left(f_{n}(s, 0), x_{n}(s)\right) d s+\int_{0}^{t}\left(h_{n}(s), x_{n}(s)\right) d s \\
& \leq \frac{1}{2} \int_{0}^{t}\left\|h_{n}(s)\right\|_{*}^{2} d s+\frac{1}{2} \int_{0}^{t}\left\|f_{n}(s, 0)\right\|_{*}^{2} d s+\int_{0}^{t}\left\|x_{n}(s)\right\|^{2} d s .
\end{aligned}
$$

So, we obtain

$$
\left|x_{n}(t)\right|^{2}+\int_{0}^{t}\left\|x_{n}(s)\right\|^{2} d s \leq C_{2}\left\{\int_{0}^{t}\left\|h_{n}(s)\right\|_{*}^{2} d s+\int_{0}^{t}\left\|f_{n}(s, 0)\right\|_{*}^{2} d s\right\},
$$

where $C_{2}$ is a constant, so that $\left\{x_{n}\right\}$ is bounded in $C([0, T] ; H) \cap L^{2}(0, T ; V)$. Therefore, $\left\{f_{n}\left(x_{n}\right)\right\}$ is bounded in $L^{2}\left(0, T ; V^{*}\right)$. By replacing them by their subsequence, we may as- 
sume $x_{n} \rightarrow x$ in $L^{2}(0, T ; V)$. By letting $n \rightarrow \infty$ in (3.4), we have

$$
x(t)+\int_{0}^{t} T(t-s)(f(s, x(s))-h(s)) d s=0
$$

Since

$$
\begin{aligned}
x_{n}^{\prime}(t) & -x^{\prime}(t)+\Lambda\left(x_{n}(t)-x(t)\right) \\
= & \left(h_{n}(t)-h(t)\right)-\left(f_{n}\left(t, x_{n}(t)\right)-f(t, x(t))\right) \\
= & \left(h_{n}(t)-h(t)\right)-\left(f_{n}\left(t, x_{n}(t)\right)-f_{n}(t, x(t))\right)-\left(f_{n}(t, x(t))-f(t, x(t))\right),
\end{aligned}
$$

from multiplying by $x_{n}(t)-x(t)$ and the monotonicity of $f_{n}$, it follows that

$$
\begin{aligned}
\frac{1}{2}\left|x_{n}(t)-x(t)\right|^{2}+\int_{0}^{t}\left\|x_{n}(s)-x(s)\right\|^{2} d s \\
\leq \frac{1}{2} \int_{0}^{t}\left\|h_{n}(s)-h(s)\right\|_{*}^{2} d s \\
\quad+\frac{1}{2} \int_{0}^{t}\left\|f_{n}(s, x(s))-f(s, x(s))\right\|_{*}^{2} d s+\int_{0}^{t}\left\|x_{n}(s)-x(s)\right\|^{2} d s .
\end{aligned}
$$

Thus, noting that $h_{n}$ and $f_{n}(t, x)$ converge strongly to $h$ and $f(t, x)$ in $L^{2}\left(0, T ; V^{*}\right)$, respectively, we see that $x$ is a solution of the equation $(L+F+\Lambda) x=h$. Finally, to prove the uniqueness of the solution, suppose that $x_{1}$ and $x_{2}$ are solutions with initial conditions $x_{01}$ and $x_{02}$ and forcing terms $h_{1}$ and $h_{2}$, respectively. Then it is easy to see that there exists $C>0$ such that

$$
\left|x_{1}(t)-x_{2}(t)\right|^{2}+\int_{0}^{t}\left\|x_{1}(s)-x_{2}(s)\right\|^{2} d s \leq C\left\{\left|x_{01}-x_{02}\right|+\int_{0}^{t}\left\|h_{1}(s)-h_{2}(s)\right\|_{*}^{2} d s\right\} .
$$

This completes the proof of Theorem 3.1.

Remark 3.1 In a similar way to Theorem 3.1, we also obtain the existence of solutions of (3.2) in the case where $f$ is a demicontinuous bounded mapping from $[0, T] \times V$ into $H$. Moreover, assume that $f(t, \cdot)$ for each $t$ is monotone as a mapping from $D(A)$ into $H$ and $x_{0} \in V, h \in L^{2}(0, T ; H)$, then there exists a unique solution $x$ of (3.1) such that

$$
x \in L^{2}(0, T ; D(A)) \cap W^{1,2}(0, T ; H) \subset C([0, T] ; V) .
$$

Moreover, there exists a constant $C_{2}$ such that

$$
\|x\|_{L^{2}(0, T ; D(A)) \cap W^{1,2}(0, T ; H)} \leq C_{2}\left(\left\|x_{0}\right\|+\|h\|_{L^{2}(0, T ; H)}\right),
$$

where $C_{2}$ is a constant depending on $T$ and the mapping

$$
V \times L^{2}(0, T ; H) \ni\left(x_{0}, h\right) \mapsto x \in L^{2}(0, T ; D(A)) \cap W^{1,2}(0, T ; H)
$$

is Lipschitz continuous. 


\section{Approximate controllability}

In this section, we deal with the approximate controllability for the semilinear equation in $H$ as follows.

$$
\left\{\begin{array}{l}
x^{\prime}(t)+A x(t)+f(t, x(t), u(t))+B u(t)=0, \quad 0<t \leq T, \\
x(0)=x_{0} .
\end{array}\right.
$$

In (4.1), the principal operator $-A$ generates an analytic semigroup $S(t)$ as stated in Section 2. Let $U$ be a Hilbert space of control variables, and let $B$ be a bounded linear operator from $U$ to $H$, which is called a controller. The mild solution of initial value problem (4.1) is the following form:

$$
x(t ; f, u)=S(t) x_{0}-\int_{0}^{t} S(t-s)\{f(s, x(s), u(s))+B u(s)\} d s .
$$

Let $f$ be a nonlinear mapping satisfying the following.

Assumption (F1) The mapping $f$ is demicontinuous bounded from $[0, T] \times H \times U$ into $V^{*}$. Assume that $f(t, \cdot, u)$ for each $(t, u) \in[0, T] \times U$ is monotone as a mapping from $V$ into $V^{*}$ with $f(t, \cdot, 0)=0$, and $f(t, x, \cdot)$ for each $(t, x) \in[0, T] \times V$ is monotone as a mapping from $U$ into $V^{*}$.

For each $u \in L^{2}(0, T ; U)$, let us define $F(t, x(t))=f(t, x(t), u(t))$. Then from Theorem 3.1 it follows that solution (4.1) exists and is unique in $L^{2}(0, T ; V) \cap W^{1,2}\left(0, T ; V^{*}\right)$. Let $x(T ; f, u)$ be a state value of system (4.1) at time $T$ corresponding to the nonlinear term $f$ and the control $u$. We define the reachable sets for system (4.1) as follows:

$$
\begin{aligned}
& R_{T}(f)=\left\{x(T ; f, u): u \in L^{2}(0, T ; U)\right\}, \\
& R_{T}(0)=\left\{x(T ; 0, u): u \in L^{2}(0, T ; U)\right\} .
\end{aligned}
$$

Definition 4.1 System (4.1) is said to be approximately controllable at time $T$ if for every desired final state $x_{1} \in H$ and $\epsilon>0$, there exists a control function $u \in L^{2}(0, T ; U)$ such that the solution $x(T ; f, u)$ of $(4.1)$ satisfies $\left|x(T ; f, u)-x_{1}\right|<\epsilon$, that is, $\overline{R_{T}(f)}=H$, where $\overline{R_{T}(f)}$ is the closure of $R_{T}(f)$ in $H$.

Definition 4.2 Let $L$ be a mapping from a Banach space $X$ into its conjugate space $X^{*}$. $T$ is called coercive if there exists $u_{0} \in D(L)$ such that

$$
\lim _{u \in D(L),\|u\| \rightarrow \infty} \frac{\left(L u, u-u_{0}\right)}{\|u\|}=\infty
$$

Remark 4.1 [23, Theorem 1.3] It is well known that if $X$ is a reflexive Banach space and $L$ is monotone, everywhere defined and hemicontinuous from $D(L)=X$ into $X^{*}$, then $L$ is maximal monotone. If in addition $L$ is coercive monotone, then $R(L)=X^{*}$.

First, we consider the approximate controllability of system (4.1) in the case where the controller $B$ is the identity operator on $H$ under Assumption (F1) on the nonlinear oper- 
ator $f$. So, $H=U$ obviously. Consider the linear system given by

$$
\left\{\begin{array}{l}
y^{\prime}(t)+A y(t)+u(t)=0, \\
y(0)=x_{0}
\end{array}\right.
$$

and the following semilinear control system:

$$
\left\{\begin{array}{l}
x^{\prime}(t)+A x(t)+f(t, x(t), v(t))+v(t)=0, \\
x(0)=x_{0} .
\end{array}\right.
$$

Lemma 4.1 Let Assumption (F1) be satisfied, and let $y(t)$ be the solution of (4.2) corresponding to a control $u$. Then there exists $v \in L^{2}(0, T ; H)$ such that

$$
\left\{\begin{array}{l}
v(t)=u(t)-f(t, y(t), v(t)), \quad 0<t \leq T, \\
v(0)=u(0) .
\end{array}\right.
$$

Proof Set

$$
w(t)=v(t)-u(t), \quad g(t, w(t))=f(t, y(t), w(t)+u(t)) .
$$

Let $(G w)(t)=g(t, w(t))$. Then equation (4.4) is equivalent to

$$
(I+G) w=0 .
$$

It is easy to see that $G$ is monotone as an operator from $L^{2}(0, T ; H)$ to $L^{2}\left(0, T ; V^{*}\right)$, and is a demicontinuous bounded mapping as an operator from $L^{2}(0, T ; H)$ into $L^{2}\left(0, T ; V^{*}\right)$. Let the collection of all finite dimensional subspaces of $H$ be denoted by $\mathcal{Y}$, and when $Y \in \mathcal{Y}$, let the orthogonal projection on $Y$ be denoted by $P_{Y}$. For $u \in L^{2}(0, T ; H)$, let us define $\left(P_{Y} u\right)(t)=P_{Y} u(t)$; thus $P_{Y}$ also denotes the orthogonal projection in $L^{2}(0, T ; H)$. According to Assumption (F1), we have that the operator $I+P_{Y} g$ is a coercive monotone operator from $Y$ into itself. In general, any demicontinuous operator is hemicontinuous. Therefore, by Remark 4.1, we have $R\left(I+P_{Y} g\right)=Y$, which (4.5) implies the existence of a solution to

$$
w_{Y}(t)+P_{Y} g\left(t, w_{Y}(t)\right)=0, \quad w_{Y}(0)=u_{Y}(0) .
$$

Since

$$
\begin{aligned}
\left|w_{Y}(t)\right|^{2} & =-\left(P_{Y} g\left(t, w_{Y}(t)\right), w_{Y}(t)\right) \\
& =-\left(P_{Y} g\left(t, w_{Y}(t)\right)-P_{Y} g(t, 0), w_{Y}(t)\right)-\left(P_{Y} g(t, 0), w_{Y}(t)\right) \\
& \leq|g(t, 0)|\left|w_{Y}(t)\right|,
\end{aligned}
$$

we have

$$
\left\|w_{Y}\right\|_{C([0, T] ; H)} \leq C .
$$


Hence, the solution of (4.6) is bounded on $C([0, T] ; H)$. Let $w$ be an arbitrary element of $L^{2}(0, T ; H)$. Then we can take $w_{n} \in Y_{n} \in \mathcal{Y}$ satisfying $(4.6)$ such that $w_{n} \rightarrow w$ in $L^{2}(0, T ; H)$. Since $G$ is monotone as an operator from $L^{2}(0, T ; H)$ to $L^{2}\left(0, T ; V^{*}\right)$, and is a demicontinuous bounded mapping, we have $G w_{n} \rightarrow G w$ in $L^{2}\left(0, T ; V^{*}\right)$. Hence, we obtain that for $v \in L^{2}(0, T ; V)$,

$$
0 \leq\left(\left(I+P_{Y} G\right) v-\left(I+P_{Y} G\right) w_{n}, v-w_{n}\right)=\left(\left(I+P_{Y} G\right) v, v-w_{n}\right)
$$

as $n \rightarrow \infty$, so that

$$
\left(\left(I+P_{Y} G\right) v, v-w\right) \geq 0 .
$$

If $v$ is replaced by $w+n^{-1} v$ in (4.7), we have

$$
0 \leq\left(w+n^{-1} v+P_{Y} G\left(w+n^{-1} v\right), n^{-1} v\right),
$$

which, by the demicontinuity of $G$, leads to

$$
0 \leq(w+G w, v), \quad \forall v \in L^{2}(0, T ; V)
$$

in the limit as $n \rightarrow \infty$. Since $v$ is arbitrary, we obtain $w+G w=0$.

Remark 4.2 As seen in [24], we know that if $X$ is a Hilbert space and $G \subset X \times X$ is maximal monotone, then $R(I+G)=X$. So, (4.5) is easily obtained if the operator $G$ in Theorem 4.1 is maximal monotone.

Theorem 4.1 Under Assumption (F1) and B = I, we have

$$
R_{T}(0) \subset R_{T}(f) .
$$

Therefore, if linear system (4.2) with $f=0$ is approximately controllable at time $T$, then so is semilinear system (4.3).

Proof Let $y, x$ be the solutions of (4.2) and (4.3), respectively. Let $v(t)=u(t)-f(t, y(t), v(t))$ in the sense of Lemma 4.1. Then, since

$$
\begin{aligned}
x^{\prime}(t) & +A x(t)+f(t, x(t), v(t))+v(t) \\
= & x^{\prime}(t)+A x(t)+f(t, x(t), v(t))+u(t)-f(t, y(t), v(t)),
\end{aligned}
$$

we have

$$
\left\{\begin{array}{l}
x^{\prime}(t)-y^{\prime}(t)+A(x(t)-y(t))+f(t, x(t), v(t))-f(t, y(t), v(t))=0, \\
x(0)-y(0)=0 .
\end{array}\right.
$$

Acting on both sides of the above equation, by $x(t)-y(t)$, from the monotonicity of $f$, it follows

$$
\frac{1}{2} \frac{d}{d t}|x(t)-y(t)|^{2}+\omega_{1}\|x(t)-y(t)\|^{2} \leq \omega_{2}|x(t)-y(t)|^{2},
$$


which is

$$
|x(t)-y(t)|^{2}+2 \omega_{1} \int_{0}^{t}\|x(s)-y(s)\|^{2} d s \leq 2 \omega_{2} \int_{0}^{t}|x(s)-y(s)|^{2} d s .
$$

By using Gronwall's inequality, we get $x=y$ in $C([0, T] ; H)$. Noting that $x(\cdot), y(\cdot) \in$ $C([0, T] ; H)$, every solution of the linear system with control $u$ is also a solution of the semilinear system with control $v$, that is, we have that $R_{T}(0) \subset R_{T}(f)$.

From now on, we consider the initial value problem for semilinear parabolic equation (4.1). Let $U$ be a Hilbert space, and let the controller operator $B$ be a nonlinear operator from $U$ to $H$.

Theorem 4.2 Let Assumption (F1) and $R(f) \subset R(B)$ be satisfied. Assume that the inverse mapping $B^{-1}$ of the controller $B$ exists and is monotone. Then the linear system

$$
\left\{\begin{array}{l}
y^{\prime}(t)+A y(t)+B u(t)=0 \\
y(0)=x_{0}
\end{array}\right.
$$

is approximately controllable at time $T$, so is nonlinear system (4.1).

Proof Let $y$ be a solution of (4.8) corresponding to a control $u$. Consider the following semilinear system:

$$
\left\{\begin{array}{l}
x^{\prime}(t)+A x(t)+f(t, x(t), v(t))+B u(t)-f(t, y(t), v(t))=0, \\
x(0)=x_{0} .
\end{array}\right.
$$

Set

$$
v(t)=u(t)-B^{-1} f(t, y(t), v(t)) .
$$

We put

$$
w(t)=v(t)-u(t), \quad\left(B_{1} u\right)(t)=B u(t), \quad(G w)(t)=g(t, w(t))=f(t, y(t), w(t)+u(t)) .
$$

Equation (4.10) is equivalent to

$$
\left(I+B_{1}^{-1} G\right) w=0
$$

Here, similarly to the proof of Lemma 4.1, we have that there exists an element $v \in$ $L^{2}(0, T ; U)$ satisfying (4.10), that is, $B v(t)=B u(t)-f(t, y(t), v(t))$. In a similar way to the proof of Theorem 4.1, we get $x=y$. Since system (4.1) is equivalent to (4.9), we conclude that $R_{T}(0) \subset R_{T}(f)$.

Now we consider the control problem of (4.1) when the controller $B$ is a nonlinear mapping in the case where $U=V$. In this case, we suppose that Assumption (F) and the next additional assumption are satisfied. 
Assumption (F2) Assume that $f(t, x, \cdot)$ for each $(t, x) \in[0, T] \times V$ is maximal monotone as a mapping from $U$ into $V^{*}$.

The following result is well known from semigroup properties.

Lemma 4.2 If $p \in L^{1}(0, T ; H)$ and

$$
\int_{0}^{t} S(t-s) p(s) d s=0, \quad 0 \leq t \leq T
$$

then $p(t)=0$ for almost all $t \in[0, T]$.

Theorem 4.3 Let Assumption (F2) and $R(f) \subset R(B)$ be satisfied. Assume that $B$ is a hemicontinuous monotone mapping from $V$ into $V^{*}$; moreover, if it is coercive, then linear system (4.8) is approximately controllable at time $T$, so is semilinear system (4.1).

Proof Let $\xi_{T} \in D(A)$. We define the linear operator $\hat{S}$ from $L^{2}(0, T ; H)$ to $H$ by

$$
\hat{S} p=\int_{0}^{T} S(T-s) p(s) d s
$$

for $p \in L^{2}(0, T ; H)$. As $\xi_{T} \in D(A)$, there exists $p \in C^{1}(0, T ; H)$ such that

$$
\hat{S} p=\xi_{T}-S(T) x_{0}
$$

for instance, take $p(s)=\left(\xi_{T}-s A \xi_{T}\right)-S(s) x_{0} / T$. By expressing $\left(B_{1} u\right)(t)=B u(t)$ for all $u \in$ $L^{2}(0, T ; V)$. By Remark 4.1, since $R(B)=V^{*}$, there exists $u_{1} \in L^{2}(0, T ; V)$ such that

$$
p=B_{1} u_{1}
$$

Since $p$ is an arbitrary element of $L^{2}\left(0, T ; V^{*}\right), p_{n} \in C^{1}(0, T ; H)$ and $p_{n} \rightarrow p$ in $L^{2}\left(0, T ; V^{*}\right)$. This implies that linear system (4.8) is approximately controllable.

To prove the approximate controllability of (4.1), we will show that $D(A) \subset \overline{R_{T}(f)}$, i.e., for given $\varepsilon>0$ and $\xi_{T} \in D(A)$, there exists $u \in L^{2}(0, T ; V)$ such that

$$
\left\|\xi_{T}-x(T ; f, u)\right\|<\varepsilon .
$$

Let $x \in L^{2}(0, T ; V)$. Then we write $G u(t)=f(t, x(t), u(t))$ for each $u \in L^{2}(0, T ; H)$. Then we rewrite (4.12) as

$$
\left\|\hat{S}\left(p+G u+B_{1} u\right)\right\|<\varepsilon .
$$

Thus, in view of Lemma 4.2, it is enough to verify that there exists an arbitrary element $u$ of $L^{2}(0, T ; V)$ such that $\left(G+B_{1}\right) u=-p$. By (2) of Lemma 2.3, $B$ is pseudo-monotone and satisfies the condition (5) of Lemma 2.3. Thus, we have $R\left(G+B_{1}\right)=V^{*}$. Since $p$ is an arbitrary element of $L^{2}\left(0, T ; V^{*}\right), p_{n} \in C^{1}(0, T ; H)$ and $p_{n} \rightarrow p$ in $L^{2}\left(0, T ; V^{*}\right)$. This implies inequality (4.3) and completes the proof of the theorem. 
Remark 4.3 We know that by Assumption (F1) and (4.8), $B_{1}+G$ is monotone, hemicontinuous and coercive from $U$ into $V^{*}$. Therefore, as seen in Remark 4.1, we have $R\left(B_{1}+G\right)=L^{2}\left(0, T ; V^{*}\right)$, that is, system (4.1) is approximately controllable.

\section{Example}

Let $\Omega$ be a bounded region in $\mathbb{R}^{n}$ with smooth boundary $\partial \Omega$. We define the following spaces:

$$
\begin{aligned}
& H^{1}(\Omega)=\left\{u: u, \frac{\partial u}{\partial x_{i}} \in L^{2}(\Omega), i=1,2, \ldots, n\right\}, \\
& H^{2}(\Omega)=\left\{u: u, \frac{\partial u}{\partial x_{i}}, \frac{\partial^{2} u}{\partial x_{i} \partial x_{j}} \in L^{2}(\Omega), i, j=1,2, \ldots, n\right\},
\end{aligned}
$$

where $\frac{\partial u}{\partial x_{i}}$ and $\frac{\partial^{2} u}{\partial x_{i} \partial x_{j}}$ are derivatives of $u$ in the distribution sense. The norm of $H^{1}(\Omega)$ is defined by

$$
\|u\|_{1}=\left\{\int_{\Omega}\left(u(x)^{2}+\sum_{i=1}^{n}\left(\frac{\partial u(x)}{\partial x_{i}}\right)^{2}\right) d x\right\}^{\frac{1}{2}} .
$$

Hence $H^{1}(\Omega)$ is a Hilbert space.

$$
H_{0}^{1}(\Omega)=\left\{u: u \in H^{1}(\Omega),\left.u\right|_{\partial \Omega}=0\right\}=\text { the closure of } C_{0}^{\infty}(\Omega) \text { in } H^{1}(\Omega) .
$$

The norm and inner product of $H_{0}^{1}(\Omega)$ are defined by

$$
\|u\|=\left\{\int_{\Omega} \sum_{i=1}^{n}\left(\frac{\partial u(x)}{\partial x_{i}}\right)^{2} d x\right\}^{\frac{1}{2}}=\|u\|_{1}, \quad((u, v))=\int_{\Omega} \sum_{i=1}^{n} \frac{\partial u(x)}{\partial x_{i}} \cdot \frac{\partial v(x)}{\partial x_{i}} d x
$$

for any $u, v \in H_{0}^{1}(\Omega)$. We put $\nabla=\left(\frac{\partial}{\partial x_{1}}, \ldots, \frac{\partial}{\partial x_{n}}\right)$. Define the operator $A$ by

$$
\begin{aligned}
& \begin{aligned}
D(A) & =\text { domain of } A \\
& =\left\{u: u \in H^{2}(\Omega) \cap H_{0}^{1}(\Omega)\right\}=\left\{u: u \in H^{2}(\Omega),\left.u\right|_{\partial \Omega}=0\right\}, \\
A u= & -\triangle u \text { for all } u \in D(A) .
\end{aligned}
\end{aligned}
$$

The operator $A$ in $L^{2}(\Omega)$ is defined so that for any $v \in H_{0}^{1}(\Omega)$, there exists $f \in L^{2}(\Omega)$ such that

$$
((u, v))=(f, v) .
$$

Then, for any $u \in D(A), A u=f$ and $A$ is a positive definite self-adjoint operator.

Let $H^{-1}(\Omega)=H_{0}^{1}(\Omega)^{*}$ be a dual space of $H_{0}^{1}(\Omega)$. For any $l \in H^{-1}(\Omega)$ and $v \in H_{0}^{1}(\Omega)$, the notation $(l, v)$ denotes the value $l$ at $v$.

Let $u$ be fixed if we consider the functional $H_{0}^{1}(\Omega) \ni v \rightarrow((u, v))$, this function is continuous linear. For any $l \in H^{-1}(\Omega)$, it follows that $(l, v)=((u, v))$. We denote that for any 
$u, v \in H_{0}^{1}(\Omega)$

$$
((u, v))=(\tilde{A} u, v)
$$

that is, $\widetilde{A} u=l$. The operator $\widetilde{A}$ is a one-to-one mapping from $H_{0}^{1}(\Omega)$ to $H^{-1}(\Omega)$. The relation of operators $A$ and $\widetilde{A}$ satisfy that

$$
\begin{aligned}
& D(A)=\left\{u \in H_{0}^{1}(\Omega), \widetilde{A} u \in L^{2}(\Omega)\right\}, \\
& A u=\widetilde{A} u \quad \text { for any } u \in D(A) .
\end{aligned}
$$

From now on, both $A$ and $\widetilde{A}$ are denoted simply by $A$.

We introduce a simple example of the control operator $B$ which satisfies the condition in Theorem 4.2. Consider the case $U=H$, and define the intercept operator $0<\alpha<T$ on $L^{2}(0, T ; H)$ by

$$
\begin{aligned}
& g_{\alpha} v(t)=\left\{\begin{array}{ll}
0, & 0 \leq t \leq \alpha, \\
|v(t)|^{p}, & p \geq 1, \alpha \leq t \leq T,
\end{array} \quad v \in L^{2}(0, T ; H),\right. \\
& B u(t)=\left(g_{\alpha} v(t)\right) v(t) .
\end{aligned}
$$

Then $B$ is a continuous monotone mapping such that there exists a constant $\beta_{1}>\beta_{2}>0$ such that

$$
\beta_{1}\left|u_{1}-u_{2}\right| \geq\left(B u_{1}-B u_{2}\right) \geq \beta_{2}\left|u_{1}-u_{2}\right|, \quad \forall u_{1}, u_{2} \in H
$$

Let

$$
H=L^{2}(\Omega), \quad V=H_{0}^{1}(\Omega), \quad V^{*}=H^{-1}(\Omega) .
$$

Let $n \geq 3$. Then by Sobolev's imbedding theorem, we have

$$
V \subset L^{p}(\Omega) \subset L^{2}(\Omega) \subset L^{q}(\Omega) \subset V^{*},
$$

where $p=2 n /(n-2)$ and $q=p /(p-1)=2 n /(n+2)$. Assume that $\hat{f}(\lambda, \mu)$ is a continuous and increasing function defined on $\mathbb{R}^{2}$ such that $|\hat{f}(\lambda, \cdot)|=O\left(|\lambda|^{(n+2) / n}\right)$ as $|\lambda| \rightarrow \infty$. If we put $f(t, x, u)(y)=f(t, x(y), u)=\hat{f}(x(y), u)$ for each $x \in H$, then $\hat{f}(\cdot, x, \cdot) \in L^{q}(\Omega)$, so that it clear that $\hat{f}$ is monotone as an operator $L^{p}(\Omega) \times U$ into $L^{q}(\Omega)$. To show that $\hat{f}$ is a demicontinuous mapping from $H$ into $L^{q}(\Omega)$, let $x_{n} \rightarrow x$ in $H$. Since $\left\{x_{n}\right\}$ is bounded in $H$, so is $\left\{\hat{f}\left(x_{n}\right)\right\}$ in $L^{q}(\Omega)$. Hence, there exists a subsequence $\left\{x_{n_{j}}\right\}$ such that $x_{n_{j}}(y) \rightarrow x(y)$ almost everywhere in $\Omega$ and there exists an element $g \in L^{q}(\Omega)$ such that $\hat{f}\left(x_{n_{j}}, \cdot\right) \rightarrow g$. Since $\hat{f}(\lambda, \cdot)$ is a continuous function of real variables $\lambda, \hat{f}\left(x_{n_{j}}(y), \cdot\right) \rightarrow \hat{f}(x(y), \cdot)$ almost everywhere in $\Omega$. Otherwise, one can find an appropriate convex combination $g_{k}=\sum_{n \geq k} \lambda_{n}^{k} \hat{f}_{n}$, where $\hat{f}_{n}(x)=\hat{f}\left(x_{n_{j}}, \cdot\right)$, which is strongly convergent to $g$ in $L^{p}(\Omega)$. This says that $g_{k}(y) \rightarrow \hat{f}(x(y), \cdot)$ for all $y$ for which $\hat{f}_{n}(x(y)) \rightarrow \hat{f}(x(y), \cdot)$. Therefore, we obtain $\hat{f}=g$, that is, $\hat{f}\left(x_{n}, \cdot\right) \rightarrow \hat{f}(x, \cdot)$. Thus, all the conditions stated in Theorem 4.2 are satisfied. Therefore, nonlinear system (4.1) with monotone operators is approximately controllable at time $T$. 


\section{Competing interests}

The authors declare that they have no competing interests.

\section{Authors' contributions}

YHK drafted the manuscript and corrected the main results, JMJ carried out the main proof of this paper, and HHR participated in its design and coordination.

\section{Author details}

${ }^{1}$ Institute of Liberal Education, Catholic University of Daegu, Daegue, 712-702, Korea. ${ }^{2}$ Department of Applied Mathematics, Pukyong National University, Busan, 608-737, Korea.

\section{Acknowledgements}

This research was supported by the Basic Science Research Program through the National Research Foundation of Korea (NRF) funded by the Ministry of Education, Science and Technology (2012-0007560).

Received: 7 August 2013 Accepted: 22 October 2013 Published: 12 Nov 2013

\section{References}

1. Di Blasio, G, Kunisch, K, Sinestrari, E: $L^{2}$-regularity for parabolic partial integrodifferential equations with delay in the highest-order derivatives. J. Math. Anal. Appl. 102, 38-57 (1984)

2. Jeong, JM: Retarded functional differential equations with $L^{1}$-valued controller. Funkc. Ekvacioj 36, 71-93 (1993)

3. Jeong, JM, Kim, JR, Ju, EY: Approximate controllability and regularity for nonlinear differential equations. Adv. Differ. Equ. 2011(27), 1-14 (2011)

4. Lions, JL: Remarks on evolution inequalities. J. Math. Soc. Jpn. 18, 331-342 (1966)

5. Stampacchia, G: Variational inequalities. In: Actes du Congrès Int. des Math., vol. 2, pp. $877-883$ (1970)

6. Browder, FE: Recent results in nonlinear functional analysis and applications to partial differential equations. In: Actes du Congrès Int. des Math. vol. 2, pp. 821-829 (1970)

7. Kenmochi, N: Nonlinear operators of monotone type in reflexive Banach spaces and nonlinear perturbations. Hiroshima Math. J. 4, 229-263 (1974)

8. Ouchi, S: On the analyticity in time of solutions of initial boundary value problems for semi-linear parabolic differential equations with monotone non-linearity. J. Fac. Sci., Univ. Tokyo, Sect. 1A, Math. 21, 19-41 (1974)

9. Ahmed, NU, Xiang, X: Existence of solutions for a class of nonlinear evolution equations with nonmonotone perturbations. Nonlinear Anal., Theory Methods Appl. 22(1), 81-89 (1994)

10. Aizicovici, S, Papageorgiou, NS: Infinite dimensional parametric optimal control problems. Jpn. J. Ind. Appl. Math. 10 307-332 (1993)

11. Hirano, N: Nonlinear evolution equations with nonmonotonic perturbations. Nonlinear Anal., Theory Methods Appl. 13(6), 599-609 (1989)

12. Pascali, D, Sburlan, S: Nonlinear Mappings of Monotone Type. Editura Academiei, Bucuresti (1978)

13. Morosanu, G: Nonlinear Evolution Equations and Applications. Reidel, Dordrecht (1988)

14. Kato, T: Nonlinear evolution equations in Banach spaces. Proc. Symp. Appl. Math. 17, 50-67 (1964)

15. Tanabe, H: Equations of Evolution. Pitman, London (1979)

16. Naito, K: Controllability of semilinear control systems dominated by the linear part. SIAM J. Control Optim. 25 715-722 (1987)

17. Jeong, JM, Ju, JE, Lee, KY: Controllability for variational inequalities of parabolic type with nonlinear perturbation. J. Inequal. Appl. 2010, Article ID 768469 (2010)

18. Sukavanam, N, Tomar, NK: Approximate controllability of semilinear delay control system. Nonlinear Funct. Anal. Appl. 12, 53-59 (2007)

19. Zhou, HX: Controllability properties of linear and semilinear abstract control systems. SIAM J. Control Optim. 22, 405-422 (1984)

20. Jeong, JM, Kwun, YC, Park, JY: Approximate controllability for semilinear retarded functional differential equations J. Dyn. Control Syst. 5(3), 329-346 (1999)

21. Lions, JL, Magenes, E: Non-Homogeneous Boundary Value Problems and Applications. Springer, Berlin (1972)

22. Triebel, H: Interpolation Theory, Function Spaces, Differential Operators. North-Holland, Amsterdam (1978)

23. Barbu, V: Nonlinear Semigroups and Differential Equations in Banach Space. Noordhoff, Leiden (1976)

24. Minty, GA: Theorem on maximal monotone sets in Hilbert space. J. Math. Anal. Appl. 11, 434-439 (1965)

10.1186/1029-242X-2013-534

Cite this article as: Kang et al.: Controllability for nonlinear evolution equations with monotone operators. Journal of Inequalities and Applications 2013, 2013:534 